\title{
SYMMETRY BREAKING IN THE SOLAR DYNAMO: NONLINEAR SOLUTIONS
}

\author{
R.L. JENNINGS \\ Department of Mathematics and Statistics \\ The University \\ Newcastle upon Tyne NE1 TRU \\ $U K$ \\ N.O. WEISS \\ Department of Applied Mathematics and Theoretical Physics \\ University of Cambridge \\ Cambridge CBS $9 E W$ \\ $U K$
}

\begin{abstract}
We examine an idealized $\alpha \omega$-dynamo model in which the magnetic fields depend only on latitude and time. The solutions that bifurcate from the fieldfree state are either symmetric or antisymmetric about the equator (quadrupolar or dipolar respectively). Nonlinear steady and periodic solutions, whether stable or unstable, can be followed numerically as the dynamo number is varied, revealing a rich bifurcation structure with mixed-mode solutions (lacking symmetry about the equator) appearing at secondary bifurcations. These results show how stable asymmetric fields can occur in the sun and illustrate the formation of complicated spatial structure in more active stars.
\end{abstract}

We assume that the generation of magnetic fields in a star like the sun occurs in a shell at the base of the convective zone and can be described by an axisymmetric mean-field $\alpha \omega$-dynamo (Parker 1979). In order to investigate latitudinal structure and equatorial symmetries we consider a highly simplified model in which only the essential physics is retained; all results are therefore strictly qualitative.

We neglect curvature and replace spherical co-ordinates $(r, \theta, \phi)$ by cartesians $(z, x, y)$ respectively. Then we may consider a magnetic field $\mathbf{B}=(0, B, \partial A / \partial x)$ that depends only on the colatitude $x$ and on time $t$. In the weak field limit the toroidal field $B$ is created by the sheared azimuthal velocity $\mathbf{u}=\omega z \sin x \hat{\mathbf{y}}$ while generation of the poloidal vector potential $A$ through helicity is represented by the parameter $\alpha=\alpha_{0} \cos x$ which is antisymmetric about the equator $(x=$ 
$\pi / 2)$. In the nonlinear regime we introduce parameters $\tau, \kappa$ and $\lambda$ to represent quenching of the $\alpha$-effect, quenching of differential rotation and enhanced losses through magnetic buoyancy respectively (Jones 1983; Weiss et al. 1984). Thus we obtain the nondimensionalised system

$$
\begin{gathered}
\partial A / \partial t=D B \cos x\left(1+\tau B^{2}\right)^{-1}+\partial^{2} A / \partial x^{2}, \\
\partial B / \partial t=(\partial A / \partial x) \sin x\left(1+\kappa B^{2}\right)^{-1}+\partial^{2} B / \partial x^{2}-\lambda B^{3} .
\end{gathered}
$$

Here the dynamo number $D=-\alpha_{0} \omega R^{3} / \eta^{2}$, where $R$ is the latitudinal length scale and $\eta$ is a turbulent diffusivity (cf. Stix 1972). The boundary conditions at the poles are $A=B=0$ at $x=0, \pi$.

Equations (1) and (2) possess a trivial field-free solution $A=B=0$. Branches that bifurcate from this trivial solution involve magnetic fields that are either symmetric (quadrupole) or antisymmetric (dipole) about the equator, with $\partial B / \partial x=$ $A=0$ or $B=\partial A / \partial x=0$ respectively at $x=\pi / 2$. For $D>0$ we expect to find antisymmetric dynamo waves migrating towards the equator but as $D$ is increased from zero the first bifurcation, at $D \approx 9$, is to a branch of steady quadrupole solutions. A branch of oscillatory dipole solutions appears at $D \approx 102$, followed by oscillatory quadrupole solutions at $D \approx 264$.

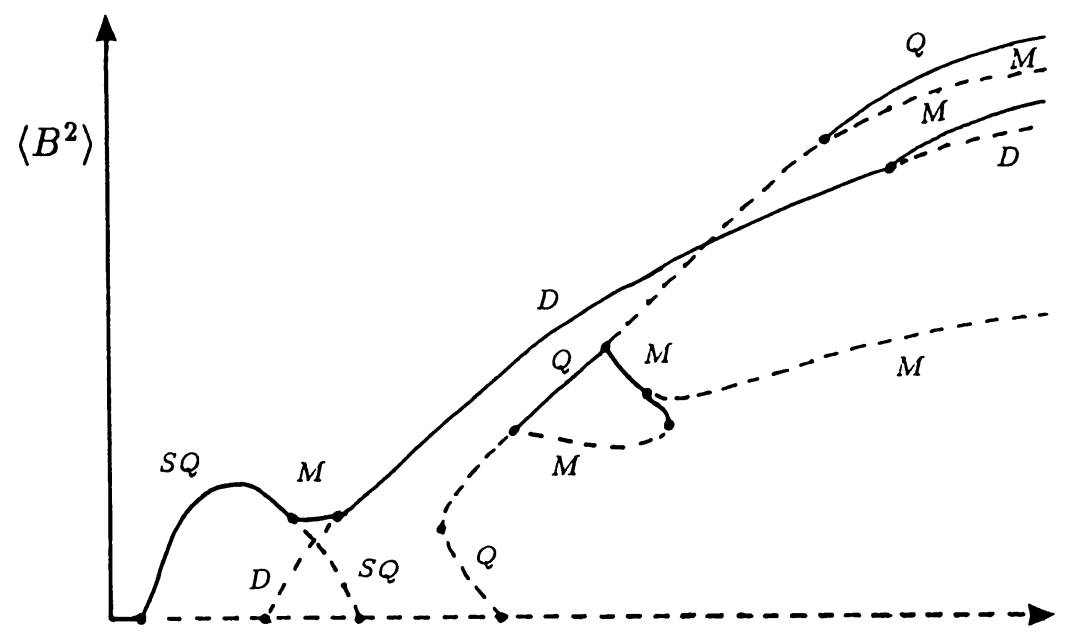

D

Figure 1. Bifurcation diagram for the case $\kappa=\lambda, \tau=0$, showing the mean square toroidal field $\left\langle B^{2}\right\rangle$ as a function of the dynamo number $D$ (not to scale). Solid (broken) lines indicate stable (unstable) solution branches. Steady quadrupole and oscillatory dipole, quadrupole and mixed-mode solutions are denoted by $S Q, D, Q$ and $M$ respectively. 
(a)

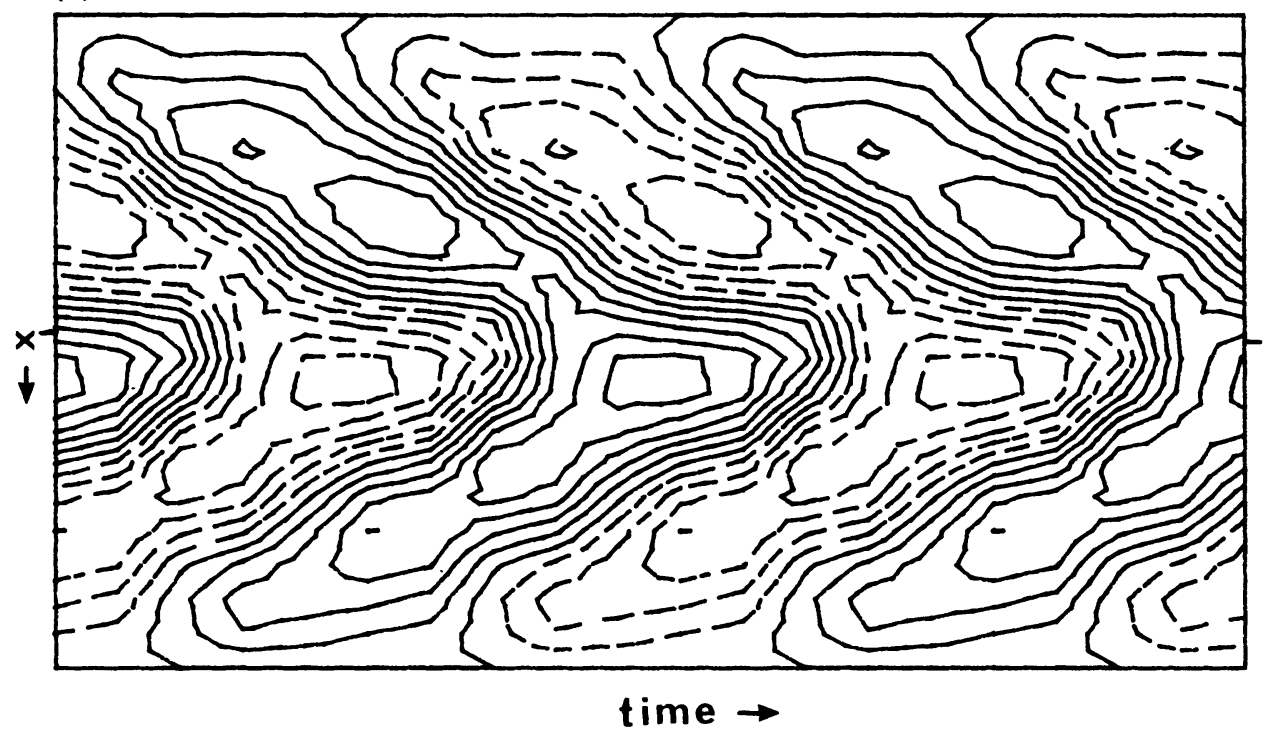

(b)

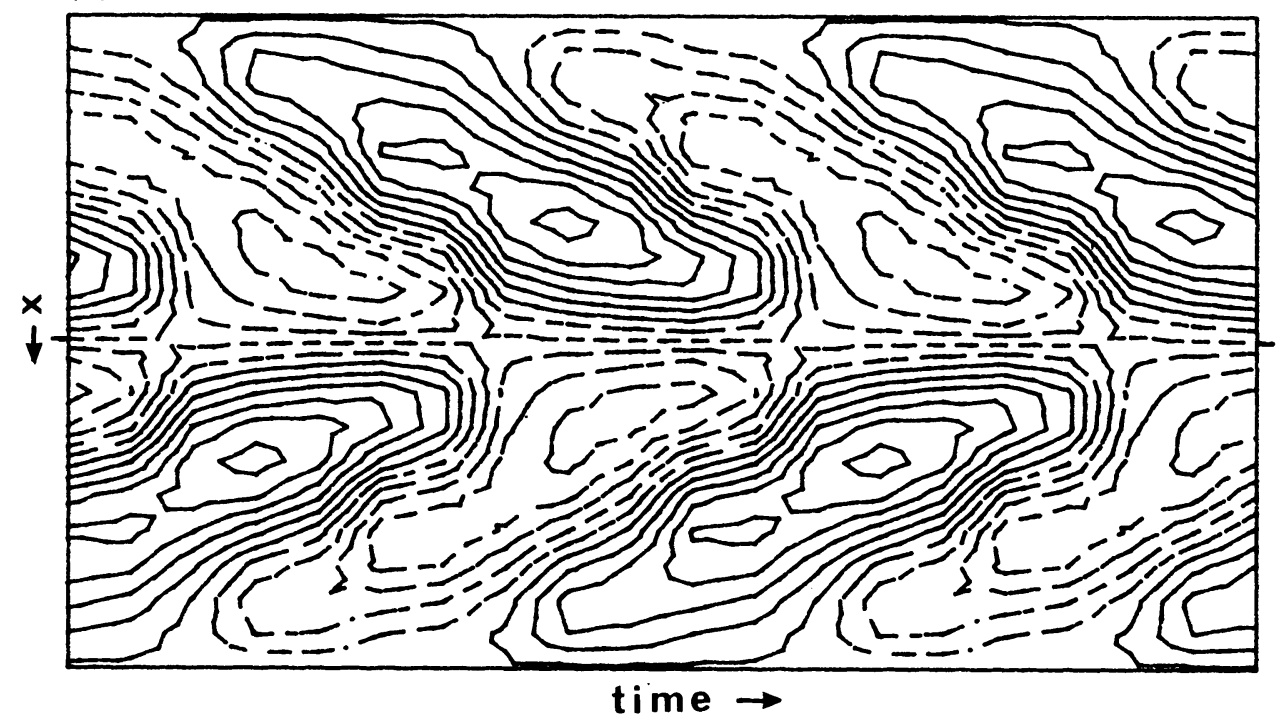

Figure 2. Butterfly diagrams with contours of the toroidal field $B(x, t)$ for mixedmode periodic solutions. Full (broken) contours indicate positive (negative) values of $B$. Both solutions obtained with a total of 14 modes at $D=2700$, when symmetry (a) is stable and symmetry (b) is unstable. 
Nonlinear behaviour can be followed by expanding $A$ and $B$ as truncated Fourier series (with a total of 30 modes). Steady or periodic solutions can then be found numerically both when they are stable and when they are unstable. Figure 1 shows the bifurcation structure when $\kappa=\lambda$ and $\tau=0$. Stability is transferred from steady quadrupole solutions to a branch of periodic mixed-mode solutions (lacking any symmetry about the equator) at $D \approx 134$ and thence to periodic dipole solutions at $D=157$. The antisymmetric dipole solutions remain stable until $D \approx 2580$ when they lose stability to mixed-mode solutions. After such a secondary bifurcation the mixed-mode solutions with period $P$ retain either the symmetry (a): $B(x, t)=-B\left(x, t+\frac{1}{2} P\right)$ or the symmetry (b): $B(x, t)=B\left(\pi-x, t+\frac{1}{2} P\right)$. These symmetries may be lost in a tertiary bifurcation. Solutions with symmetry (a) only have fields that are consistently stronger in one hemisphere than in the other, while those with symmetry (b) have persistent fields of the same sign at the equator. Figure 2 shows examples of butterfly diagrams for both cases.

The branch of oscillatory quadrupole solutions is initially unstable but finally gains stability at $D \approx 2335$. For a range of parameter values there are then two stable periodic solutions with different basins of attraction as well as a number of unstable solutions with different symmetries. At larger values of $D$ there are still more bifurcations whose details depend on the number of terms included in the series.

These results demonstrate that linear theory gives a qualitatively misleading picture of behaviour in the nonlinear regime (cf. Brandenburg et al. 1989; Schmitt $\&$ Schüssler 1989). Stable solutions are determined by a complicated bifurcation structure like that illustrated in Figure 1. For small $D$ there exist pure quadrupole and pure dipole solutions as well as mixed-mode solutions resembling behaviour observed in the sun, where a $10 \%$ asymmetry in magnetic flux has persisted over several cycles (Tang et al. 1984). As $D$ increases subsidiary bifurcations lead to multiple solutions with richer spatial structure. Moreover, dynamical effects can produce chaotic time dependence (Weiss et al. 1984). We should therefore expect to find complicated spatiotemporal patterns of activity in rapidly rotating stars.

\section{REFERENCES}

Brandenburg, A., Krause, F., Meinel, R., Moss, D. \& Tuominen, I. 1989. Astron. Astrophys., in press.

Jones, C.A. 1983. In Stellar and Planetary Magnetism (ed. A.M. Soward), p. 159. Gordon \& Breach, London.

Parker, E.N. 1979. Cosmical Magnetic Fields. Oxford University Press.

Schmitt, D. \& Schüssler, M. 1989. Astron. Astrophys., submitted.

Stix, M. 1972. Astron. Astrophys. 20, 9.

Tang, F., Howard, R. \& Adkins, J.M. 1984. Solar Phys. 91, 75.

Weiss, N.O., Cattaneo, F. \& Jones, C.A. 1984. Geophys. Astrophys. Fluid Dyn. 30, 305. 